\title{
The emerging challenge of pain in systemic sclerosis: Similarity to the pain experience reported by Sjőgren's syndrome patients
}

\author{
Rossella De Luca ${ }^{1}$, Marco Maresca ${ }^{1, *}$, Martina Orlandi ${ }^{1}$, Cosimo Bruni ${ }^{1}$, Kamal Solanki $^{1,2}$, Yukai Wang ${ }^{1,3}$, Gemma Lepri ${ }^{1}$ \\ Serena Guiducci ${ }^{1}$, Daniela Melchiorre ${ }^{1}$, Alberto Moggi-Pignone ${ }^{1}$, Wanda Rossi ${ }^{4}$, Amato De Paulis ${ }^{4}$, Marta Dzhus ${ }^{1,5}$, \\ Marco Matucci-Cerinic ${ }^{1,6}$, Silvia Bellando-Randone ${ }^{1}$ \\ ${ }^{1}$ Department of Experimental and Clinical Medicine, University of Florence, \& Division of Rheumatology AOUC, Florence, Italy \\ ${ }^{2}$ Department of Rheumatology, Waikato Hospital, Hamilton, New Zealand \\ 3Department of Rheumatology and Immunology, Shantou Central Hospital, Shantou, Guangdong Province, China \\ ${ }^{4}$ Department of Translational Medical Sciences, University Federico II, Naples, Italy \\ ${ }^{5}$ Department of Internal Medicine, O. Bogomolets National Medical University, Kyiv, Ukraine \\ ${ }^{6}$ Unit of Immunology, Rheumatology, Allergy and Rare Diseases (UnIRAR), IRCCS San Raffaele Hospital, Milan, Italy
}

\begin{abstract}
In order to evaluate the importance of pain in systemic sclerosis (SSc), the characteristics of pain reported by patients with SSc were analyzed and compared with the characteristics of pain reported by patients with primary Sjögren's syndrome (pSS). Pain was reported by 56 patients (80\%) in a group of 70 patients with SSc and by 25 patients (78\%) in a group of 32 patients with pSS. Pain severity was assessed by the Pain Rating Index (PRI) and the Present Pain Intensity (PPI) of the McGill Pain Questionnaire (MPQ) and by values obtained by a visual analog scale (VAS) indicating the intensity of pain felt in the moment of the examination and the intensity of pain felt in the week preceding the moment of the examination. No significant difference was detected in the comparison of mean values of pain indices between patients with SSc and patients with pSS and in the comparison among subgroups of patients with SSc. The data indicate that pain is a frequent and important cause of suffering in SSc as in other chronic diseases. The association of different methods may be especially useful to obtain a careful evaluation of pain in clinical research.
\end{abstract}

Received February 1, 2021 accepted July 25, 2021

Keywords

digital ulcers $\bullet$ joint pain $•$ pain assessment $•$ primary Sjögren's syndrome $\bullet$ systemic sclerosis

\section{Introduction}

Systemic sclerosis (SSc) is a connective autoimmune disease with a complex pathophysiology: ${ }_{i}^{[1]}$ In this disease, pain has not been considered up to now as a meaningful issue, in particular when it is compared to clinical features that usually jeopardize patients' survival. However, pain is frequently reported by SSc patients and may play an important role in provoking suffering and disability. ${ }^{[2-8]}$ In fact, pain was reported by most SSc patients (63\%) in a sample including 142 patients observed in the study by Benrud-Larson et al. ${ }^{[2]}$ In another cohort, $75 \%$ of SSc patients experienced pain since the onset of the disease. ${ }^{[3]}$ Moreover, a high percentage $(83 \%)$ of patients reporting pain was also observed in a large sample including $585 \mathrm{SSc}$ patients by Schieir et al. ${ }^{[6]}$ Among 537 European patients with SSc from 5 European countries examined by Willems et al., ${ }^{[7]}$ joint pain and muscle pain were experienced by at least $70 \%$ of patients in all 5 countries. In a group of 42 SSc patients examined by Ostojic et al., ${ }^{[8]}$ $92.9 \%$ of patients were suffering from different types of pain. In SSc, pain has a remarkable impact on quality of life..$^{[2,7,8]}$ A high correlation between pain intensity and scores evaluating altered quality of life has been also reported..$^{[5]}$ In SSc, 
pain is frequently due to skin ulcers ${ }^{[8-13]}$ and/or to the involvement of musculoskeletal system. ${ }^{[8,14]}$ Arthritis is a common source of pain. ${ }^{7,8,14-17]}$

In another connective autoimmune disease like primary Sjögren's syndrome (pSS), the problem of pain is significant and needs to be addressed very frequently in practice. ${ }^{[18]}$ In order to evaluate the relevance of pain in SSc patients, the present study investigated the characteristics of pain compared with those reported by patients affected by another connective autoimmune disease like pSS.

The assessment of pain is a crucial problem in clinical studies on pain syndromes. It was greatly improved by the introduction of 2 methods: the use of the "McGill Pain Questionnaire" (MPQ) ${ }^{[19]}$ and the use of visual analog scale (VAS) for the evaluation of pain intensity ${ }^{[20]}$. Both methods have been widely used in clinical investigations on different pain syndromes. ${ }^{[21]}$

In chronic diseases, the intensity of pain may change in different days and in different moments of the day. As a consequence, the assessment of pain intensity in the moment in which a patient is observed by a physician is not always a useful index of pain severity. In clinical examination of a patient experiencing pain, it may be useful to ask the patient not only to report pain intensity in the very moment of the examination but also to report an evaluation of pain intensity during a period preceding the examination. In the present study, pain was assessed by VAS and by the MPQ. VAS was used to evaluate pain intensity in the moment of the examination and in the preceding week.

\section{Materials and Methods}

The investigation was performed in the Division of Rheumatology of the University Hospital of the University of Florence. The occurrence of pain was assessed in a group of 70 consecutive SSc patients (64 women and 6 men, with age ranging from 21 years to 84 years, mean age \pm standard deviation (SD): $57.17 \pm 16.66$ years) and in a group of 32 consecutive pSS patients ( 29 women and 3 men, with age ranging from 34 years to 82 years, mean age $\pm S D$ : $60.22 \pm 11.71$ years). Patients were classified according to American College of Rheumatology criteria for $\mathrm{SSc}^{[22]}$ and for pSS. ${ }^{[23]}$ The investigation was approved by the Ethical Committee of the Tuscan Region (10.7.2017, approval number 11027) and informed consent was obtained by every patient.

Patients with SSc were divided into limited SSc (ISSc) and diffuse SSc (dSSc) groups according to the classification of LeRoy et al. ${ }^{[24]}$ In the whole group of patients with SSc reporting pain, 3 subgroups of patients were identified, with different clinical features that could be related to different pain producing mechanisms: (1) patients with pain due to digital ulcers; (2) patients with joint pain; and (3) patients reporting widespread pain with the typical characteristics of fibromyalgia. ${ }^{[25]}$ Joint pain was present in all the patients with pSS reporting pain: no subgroups could be identified. No patient reported the use of analgesic drugs in the week preceding the moment of the examination. Patients were asked to report whether they felt pain in any part of the body. In every patient reporting pain, the characteristics of pain were analyzed by the MPQ and by VAS. The Italian version of the MPQ proposed by Maiani and Sanavio ${ }^{[26]}$ was used. VAS was a $10-\mathrm{cm}$ vertical segment with the indications "no pain" at the lower extremity and "unbearable pain" at the upper extremity: the patient was required to indicate the point corresponding to the intensity of his/her pain; the measure of pain intensity was the number of millimeters measuring the length from the lower extremity of the scale to the point indicated by the patient. Every patient was asked to indicate by VAS the intensity of pain felt in the moment of the examination and the intensity of pain felt during the week preceding the moment of the examination.

The following indices of pain severity were analyzed:

- the Pain Rating Index (PRI), calculated by the analysis of the terms chosen by the patient in the list of verbal descriptors of the MPQ;

- the Present Pain Intensity (PPI), obtained by the verbal rating scale included in the MPQ;

- the value obtained by VAS indicating the intensity of pain in the moment of the examination; and

- the value obtained by VAS indicating the intensity of pain felt during the week preceding the moment of the examination.

The mean values of indices calculated in patients with SSc were compared with the mean values calculated in patients with pSS. The means of indices calculated in patients with ISSc were compared with the means of indices in patients with dSSc. The means of indices calculated in every subgroup of patients with SSc were compared with the means of the other subgroups, with the means of the whole group of patients with SSc reporting pain, and with the means of the group of patients with pSS reporting pain. Comparisons of means were performed by Student's $t$ test.

\section{Results}

Pain was reported by 56 patients with SSc (80\%) and by 25 patients with Sjögren's syndrome (78\%).

The group of 56 patients with SSc reporting pain included 52 women and 4 men, with age ranging from 21 years to 84 years (mean age \pm SD: $59.05 \pm 16.27$ years). No correlation was 
observed between the occurrence of pain and the duration of the disease, autoantibodies, or the presence of visceral involvement. ISSc was present in 35 patients (33 women and 2 men, with age ranging from 21 years to 84 years and mean age \pm SD: $59.63 \pm 17.37$ years), and dSSc was present in 21 patients ( 19 women and 2 men, with age ranging from 34 years to 80 years and mean age \pm SD: $57.48 \pm 14.17$ years). Fourteen women, with ages ranging from 24 years to 83 years and a mean age $( \pm S D)$ of $54.64( \pm 18.35)$ years, reported pain due to digital ulcers; 27 patients, 23 women and 4 men, with ages ranging from 21 years to 81 years and a mean age $( \pm S D)$ of $56.65( \pm 17.12)$ years, reported joint pain; 15 women, with ages ranging from 38 years to 84 years and a mean age $( \pm S D)$ of $63.33( \pm 13.97)$ years, reported widespread pain with the typical characteristics of fibromyalgia. ${ }^{[25]}$ The importance of neuropathic pain in SSc has been recently considered. ${ }^{[27-29]}$ Among the patients with SSc and pain examined in the present investigation, in 2 patients with joint pain and in 1 patient with pain due to digital ulcers, some clinical features were present which could suggest a component of neuropathic pain: those patients reported a "burning" quality of spontaneous pain associated with paresthesias in some body regions, and in the same regions, the phenomenon of hyperalgesia was observed at sensory examination. No patient reported previous herpes zoster virus infection.

The group of 25 patients with pSS reporting pain included 23 women and 2 men, with age ranging from 34 years to 82 years (mean age \pm SD: $59.12 \pm 12.16$ years). In all patients, pain was due to joint involvement. No correlation was observed between the occurrence of pain and the duration of the disease or autoantibodies (SSA and SSB). A component of neuropathic pain could be concomitant to joint pain in 4 patients.

Mean values $( \pm S D)$ of the different pain indices of patients with SSc and of patients with pSS and $P$ values calculated in the comparison of means of the 2 groups of patients are reported in Table 1. No significant difference was observed.

\section{Patients with SSc: Comparison of the Means of the Indi- ces Obtained in Patients with ISSc and in Patients with dSSc}

The means of the indices of patients with ISSc and dSSc and $P$ values calculated in the comparison of means are reported in Table 2. The mean of the PRI of patients with ISSc was significantly higher than that of dSSc patients $(P<0.05)$. The differences of the means of the other indices were not statistically significant.

\section{Characteristics of Pain in Different Subgroups of Patients with SSc}

1) Patients with pain due to digital ulcers (14 patients).

The patients of this subgroup reported pain with the typical characteristics of cutaneous pain, i.e., pain well localized and well defined; hyperalgesia was evident when mechanical stimuli were applied on the ulcers and on the surrounding areas, with pain often accompanied by a strong reaction. The terms most frequently chosen in the MPQ by the patients of this subgroup to describe their pain were corresponding to the English terms "pricking," "boring," and "smarting."

2) Patients with joint pain (27 patients).

Signs and symptoms of arthritis were evident in 8 patients; tenosynovitis was present in 4 patients (confirmed at ultrasound examination). In the other 15 patients of this subgroup, no signs of joint inflammation could be detected by ultrasound or radiologic examination or laboratory tests. The patients of this subgroup reported pain with the typical characteristics of deep somatic pain, i.e., deep pain usually well localized but often radiating from the site of origin; deep tenderness was observed: pain was evoked either by the application of pressure or by passive movement of the affected joints. The terms most frequently chosen in the MPQ by the patients of this subgroup to describe their pain were corresponding to the English terms "gnawing," "cramping," and "aching."

Table 1: Comparison of the mean values of pain indices obtained in patients with systemic sclerosis with the mean values of pain indices obtained in patients with primary Sjögren's syndrome (Student's t test)

\begin{tabular}{|c|c|c|c|}
\hline Pain indices & $\begin{array}{l}\text { Systemic sclerosis } \\
\text { Mean ( } \pm \text { SD) }\end{array}$ & $\begin{array}{c}\text { Primary Sjőgren's syndrome } \\
\text { Mean }( \pm S D)\end{array}$ & $P$ values \\
\hline PRI (Pain Rating Index) & $15.25( \pm 12.64)$ & $14.20( \pm 9.34)$ & 0.964 \\
\hline PPI (Present Pain Intensity) & $2.76( \pm 1.07)$ & $2.48( \pm 0.77)$ & 0.539 \\
\hline VAS value indicating pain intensity in the moment of the examination & $44.48( \pm 26.91)$ & $50.21( \pm 21.39)$ & 0.906 \\
\hline VAS value indicating pain intensity in the preceding week & $50.38( \pm 24.20)$ & $45.88( \pm 19.62)$ & 0.079 \\
\hline
\end{tabular}

Of 56 patients with SSc, 52 were women and four were men, with age ranging from 21 years to 84 years (mean age \pm SD: $59.05 \pm 16.27$ years); of 25 patients with pSS, 23 were women and two were men, with age ranging from 34 years to 82 years (mean age \pm SD: $59.12 \pm 12.16$ years). VAS, visual analog scale. 
Table 2: Comparison of the mean values of pain indices of patients with limited systemic sclerosis with the mean values of patients with diffuse systemic sclerosis (Student's t test)

\begin{tabular}{|c|c|c|c|}
\hline Pain indices & $\begin{array}{l}\text { Patients with limited systemic sclerosis } \\
\text { Mean }( \pm \text { SD) }\end{array}$ & $\begin{array}{l}\text { Patients with diffuse systemic sclerosis } \\
\text { Mean }( \pm \text { SD) }\end{array}$ & $P$ values \\
\hline PRI (Pain Rating Index) & $18.63( \pm 13.88)$ & $11.90( \pm 8.04)$ & 0.048 \\
\hline PPI (Present Pain Intensity) & $2.90( \pm 1.06)$ & $2.36( \pm 1.03)$ & 0.153 \\
\hline $\begin{array}{l}\text { VAS value indicating pain intensity in the moment } \\
\text { of the examination }\end{array}$ & $44.26( \pm 27.64)$ & $44.86( \pm 26.32)$ & 0.932 \\
\hline VAS value indicating pain intensity in the preceding week & $50.49( \pm 25.96)$ & $49.24( \pm 20.58)$ & 0.852 \\
\hline
\end{tabular}

Of 35 patients with ISSc, 33 were women and two were men, with age ranging from 21 years to 84 years (mean age \pm SD: $59.63 \pm 17.37$ years); of 21 patients with dSSc, 19 were women and two were men, with age ranging from 34 years to 80 years (mean age \pm SD: $57.48 \pm 14.17$ years). VAS, visual analog scale.

Table 3: Means $( \pm S D)$ of pain indices in the different subgroups of patients with systemic sclerosis and pain

\begin{tabular}{lccc}
\hline Pain indices & $\begin{array}{c}\text { Patients with digital ulcers } \\
\text { Mean }( \pm \text { SD })\end{array}$ & $\begin{array}{c}\text { Patients with joint pain } \\
\text { Mean }( \pm \text { SD })\end{array}$ & $\begin{array}{c}\text { Patients with widespread pain } \\
\text { Mean }( \pm \text { SD) }\end{array}$ \\
\hline PRI (Pain Rating Index) & $16.00( \pm 8.30)$ & $17.54( \pm 6.46)$ & $13.67( \pm 6.44)$ \\
PPI (Present Pain Intensity) & $2.02( \pm 0.98)$ & $2.71( \pm 1.10)$ & $3.40( \pm 1.34)$ \\
$\begin{array}{l}\text { VAS value indicating pain intensity in the moment } \\
\text { of the examination }\end{array}$ & $55.2( \pm 23.1)$ & $41.7( \pm 28.0)$ & $49.1( \pm 34)$ \\
VAS value indicating pain intensity in the preceding week & $56.1( \pm 26.3)$ & $44.8( \pm 25.6)$ & $61.7( \pm 23.4)$ \\
\hline
\end{tabular}

Fourteen women reported pain due to digital ulcers, with age ranging from 24 years to 83 years and a mean age ( \pm SD) of 54.64 ( \pm 18.35 ) years; of 27 patients with joint pain, 23 were women and four were men, with age ranging from 21 years to 81 years and a mean age ( \pm SD) of 56.65 ( \pm 17.12 ) years; 15 women reported widespread pain, with age ranging from 38 years to 84 years and a mean age $( \pm S D)$ of $63.33( \pm 13.97)$ years. VAS, visual analog scale.

3) Patients with widespread pain (15 patients).

The patients of this subgroup reported widespread pain with the typical characteristics of fibromyalgia. The terms most frequently chosen in the MPQ by the patients of this subgroup to describe their pain were corresponding to the English terms "gnawing," "pulling," and "splitting."

The means of pain indices of the different subgroups of patients are reported in Table 3.

\section{Characteristics of Pain in the Group of Patients with pSS and Pain}

All the patients of this group reported joint pain with the typical characteristics of deep somatic pain, accompanied by deep tenderness. The terms most frequently chosen in the MPQ by the patients of this subgroup to describe their pain were corresponding to the English terms "cramping," "sore," and "aching."

Comparisons of the Indices of Every Subgroup of Patients with SSc and Pain with the Indices of the other Subgroups, of the Whole Group of Patients with SSc and Pain, and of the Group of Patients with pSS and Pain

No significant difference was observed in the comparison of the means of the indices of every subgroup of patients with SSc and pain with the means of the indices of the other subgroups, in the comparison of the means of the indices of every subgroup with the means of the whole group of patients with SSc and pain, and in the comparison of the means of the indices of every subgroup with the means of the group of patients with pSS and pain.

\section{Discussion}

In the present study, a high frequency of pain (80\%) was observed in SSc patients. This finding is in agreement with the results obtained in previous investigations in which the frequency of pain in SSc was estimated to range between $63 \%$ and $92.9 \% .^{[2,3,6-8]}$ It is interesting to observe that the frequency of pain in patients with SSc was similar to the frequency observed in patients with pSS, in which pain due to joint involvement is clearly predominant. In both diseases, pain severity was assessed by four numerical indices: the $P R I$ and PPI obtained by the MPQ, a value obtained by VAS indicating pain intensity in the moment of the examination, and a value obtained by VAS indicating pain intensity in the week preceding the moment of the examination. This method of evaluation, including different indices of pain severity, was not used in previous investigation on the occurrence of pain in patients with SSc and was chosen because by this method more information may be obtained on the characteristics of pain than by a single index. 
In clinical practice, pain intensity is frequently assessed only by VAS. The use of VAS allows a quick assessment of pain intensity, which may be easily repeated even many times in the same day. The assessment of pain intensity by VAS is especially useful in the evaluation of acute pain, as in the case of post-operative pain, when the assessment of pain intensity must be repeated many times in a brief period. SSc and pSS are chronic diseases, and patients are usually monitored by periodic examinations: the value of pain intensity obtained by VAS in the moment of the examination is not always a good index because pain intensity may change even within a wide range in different days and even in the same day. In chronic pain syndromes, it may be useful to ask the patients to indicate by VAS not only the intensity of pain they feel in the moment of the examination but also the intensity of pain they felt in a brief period preceding the moment of the examination. It is important to ask the patients to indicate pain intensity with reference to a brief period of time (e.g., a week or 10 days), in which most patients may easily remember pain intensity. In the present investigation, the patients were asked to indicate pain intensity felt in the week preceding the moment of the examination. This procedure allows a better evaluation of pain.

The PPI of the MPQ is another index of pain intensity, which is obtained by a verbal rating scale. In the present investigation, the PPI was used because it is obtained by a simple procedure easily understood and easily performed by every patient, whereas the assessment of pain intensity by VAS may be difficult for some patients, who do not understand the procedure well. The association of VAS and of a verbal rating scale may be useful in clinical practice to obtain a better evaluation of pain intensity.

The PRI of the MPQ is instead an index which is different from the PPI and from values of pain intensity obtained by VAS. It is calculated by the analysis of verbal pain descriptors indicating specific characteristics of pain sensation ("quality" of pain). The value of the PRI is derived from the number of verbal descriptors chosen in the different subclasses of the MPQ (corresponding to different "qualities" of pain) and by the degree of pain severity indicated by the verbal descriptor chosen in every subclass. In the present investigation, the association of the PRI to the PPI and to values obtained by VAS could provide more information on the severity of pain.

The comparison of mean values of the different pain indices did not reveal significant differences between values obtained in the patients with SSc and pSS. This may suggest that not only the frequency but also the severity of pain is similar in patients with SSc and those with pSS.

In the group of patients with SSc and pain, 3 subgroups were identified with different clinical features: (1) patients with pain due to digital ulcers; (2) patients with joint pain; and (3) patients with widespread pain. The patients with pain due to digital ulcers were easily identified, as at each visit they could complain to the nurse about their pain. It is well known that the presence of ulcer pain is usually linked to a significant infection of the wound. Therefore, when pain is reported in an SSc ulcer, it is a very important symptom which should immediately alert the physician to investigate the presence of an infection.

In pSS, the source of pain seems to be rather different with respect to SSc. In fact, it is primarily due to joint and muscle pain, which is commonly observed in pSS where it is mainly linked to a synovitis of large and small joints, whereas in SSc, it is primarily due also to the involvement of tendons and sheaths. ${ }^{[30]}$ It is interesting to note that, in the present study, no significant difference was observed in the comparison between the pain indices of SSc patients with joint pain and the pain indices of pSS patients, i.e., between groups in which joint pain was present, even if joint pain was due to different diseases and to the involvement of different structures.

In the present study, a subgroup of SSc patients was identified in which widespread pain was present, with the characteristics of pain observed in fibromyalgia. This finding is especially interesting because the onset of widespread pain has also been reported in other chronic pain conditions, such as rheumatoid arthritis, endometriosis, and inflammatory bowel disease and has been considered consistent with a hypothesis of central sensitization as an effect of a painful underlying condition. ${ }^{[31]}$

The results of the present investigation indicate that a careful assessment of pain may be obtained by the association of different methods. The assessment of pain intensity by two different methods is especially useful because some procedures (as the use of VAS) may be difficult for some patients and other procedures (as the use of a verbal rating scale) are easy but do not allow the evaluation of slight differences. Moreover, the assessment of pain intensity in the moment of the examination of a patient should be associated with an evaluation of pain intensity during a certain period because great variations of pain intensity in different moments may occur in some patients. The assessment of pain intensity should also be associated with an assessment of pain severity obtained by a different method, such as the analysis of verbal pain descriptors chosen by the patient in the list included in the MPQ.

Recently, it has been shown that $92.9 \%$ of SSc patients suffer from different types of pain, and among them, 45.2\% complain of daily pain. ${ }^{[32]}$ In line with our data, joint pain was very frequent $(78.6 \%)$ and was followed by ischemic pain of Raynaud's phenomenon (69\%), as well as back pain 
$(47.6 \%)$, headache $(31 \%)$, chest pain $(23.8 \%)$, and odynophagia $(21.4 \%)$. To be specifically noted is the fact that they also found painful digital ulcers (19\%). Symptoms of neuropathic pain were noticed in $26.2 \%$ of patients. Pain related to Raynaud's phenomenon, digital ulcers, odynophagia, and joint pain were associated with significant symptoms of depression. Severe joint pain, everyday pain, and symptoms of neuropathic pain in SSc were associated with more severe disease and poorer quality of life. Pain is associated with more severe disease, depression, and poor quality of life. Therefore, SSc joint involvement is an area of interest, as it has been shown in a specific area by the analysis of the profile of pain threshold at the temporomandibular joint and masseter muscle level in SSc. ${ }^{[33]}$

It may be concluded that pain is a frequent and important cause of suffering and disability in SSc as in other chronic diseases. Therefore, the assessment of pain is an important part of the clinical evaluation of every patient with SSc. The association of different methods for assessing pain severity may be especially useful to obtain a careful evaluation of pain in clinical research on SSc and on other chronic diseases.

Ethical Statement

The authors declare that ethical approval were obtained and all methods were carried out in accordance with relevant guidelines and regulations.

Conflict of Interest

Marco Matucci-Cerinic is an Associate Editor-in-Chief of the journal. The article was subject to the journal's standard procedures, with peer review handled independently of this editor and his research groups.

References

[1] Varga J, Trojanowska M, Kuwana M. Pathogenesis of Systemic Sclerosis: Recent Insights of Molecular and Cellular Mechanisms and Therapeutic Opportunities. J Scleroderma Relat Disord. 2017; 2:137-152. doi: 10.5301/jsrd.5000249.

[2] Benrud-Larson LM, Haythornthwaite JA, Heinberg LJ, et al. The Impact of Pain and Symptoms of Depression in Scleroderma. Pain. 2002;95:267-275.

[3] Richards H, Herrick AL, Griffin K, et al. Systemic Sclerosis: Patients' Perception of their Condition. Arthritis Rheum. 2003;49:689-696.

[4] Carreira PE. 'Quality of Pain' in Systemic Sclerosis. Rheumatology. 2006;45:1185-1198.

[5] Georges C, Chassany O, Toledano C, et al. Impact of Pain in Health Related Quality of Life of Patients with Systemic Sclerosis. Rheumatology. 2006;45:1298-1302.

[6] Schieir O, et al. Prevalence, Severity, and Clinical Correlates of Pain in Patients with Systemic Sclerosis. Arthritis Care Res (Hoboken). 2010;62:409-417. doi: 10.1002/acc.20108.

[7] Willems LM, Kwakkenbos L, Leite CC, et al. Frequency and Impact of Disease Symptoms Experienced by Patients with Systemic Sclerosis from FIVE EUROPEAN Countries. Clin Exp Rheumatol. 2014;32(Suppl. 86):S88-S93.

[8] Ostojic P, Jankovic K, Djurovic N, et al. Common Causes of Pain in Systemic Sclerosis: Frequency, Severity, and Relationship to Disease Status, Depression and Quality of Life. Pain Manag Nurs.
2019;20:331-336. doi: 10.1016/j.pmn.2019.02.006.

[9] Di Franco M, Bazzichi L, Casale R, et al. Pain in Systemic Connective Tissue Diseases. Best Pract Res Clin Rheumatol. 2015;29:53-62. doi: 10.1016/j.berh.2015.05.006.

[10] Hachulla E, Clerson P, Launay D, et al. Natural History of Ischemic Digital Ulcers in Systemic Sclerosis: Single-Center Retrospective Longitudinal Study. J Rheumatol. 2007;34:2423-2430.

[11] Steen V, Denton CP, Pope JE, et al. Digital Ulcers: Overt Vascular Disease in Systemic Sclerosis. Rheumatology. 2009;48(Suppl. 3): iii19-iii24. doi: 10.1093/rheumatology/kep105.

[12] Amanzi L, Braschi F, Fiori G, et al. Digital Ulcers in Scleroderma: Staging, Characteristics and Sub-Setting through Observation of 1614 Digital Lesions. Rheumatology. 2010;49:1374-1382. doi: 10.1093/rheumatology/keq097.

[13] Galluccio F, Matucci-Cerinic M. Two Faces of the Same Coin: Raynaud Phenomenon and Digital Ulcers in Systemic Sclerosis. Autoimmun. Rev. 2011;10:241-243. doi: 10.1016/j.autrev.2010.09.008. [14] Clements PJ, Allanore Y, Khanna D, et al. Arthritis in Systemic Sclerosis: Systematic Review of the Literature and Suggestions for the Performance of Future Clinical Trials in Systemic Sclerosis Arthritis. Semin Arthritis Rheum. 2012;41:801-814. doi: 10.1016/j.semarthrit.2011.10.003.

[15] Avouac J, Walker U, Tyndall A, et al. Characteristics of Joint Involvement and Relationships with Systemic Inflammation in Systemic Sclerosis: Results from the EULAR Scleroderma Trial and Research 
Group (EUSTAR) Database. J Rheumatol. 2010;37:1488-1501. doi: 10.3899/jrheum.091165.

[16] Avouac J, Clements PJ, Khanna D, et al. Articular Involvement in Systemic Sclerosis. Rheumatology. 2012;51:1347-1358. doi: 10.1093/rheumatology/kes041.

[17] Schmeiser T, Pons-Kühnemann J, Ozden F, et al. Arthritis in Patients with Systemic Sclerosis. Eur J Intern Med. 2012;23:25-29. doi: 10.1016/j.ejim.2011.09.010.

[18] Vitali, C., Del Papa, N. Pain in Primary Sjögren's Syndrome. Best Pract Res Clin Rheumatol. 2015;29:63-70. doi: 10.1016/j. berh.2015.05.002.

[19] Melzack R. The McGill Pain Questionnaire: Major Properties and Scoring Methods. Pain. 1975;1:275-299.

[20] Scott J, Huskisson EC. Graphic Representation of Pain. Pain. 1976;2:175-184.

[21] Melzack R, Katz J. Pain Assessment in Adult Patients. In: McMahon SB, Koltzenburg M, eds. Wall and Melzack's Textbook of Pain, 5th edn. Elsevier Churchill Livingstone, 2006:291-304.

[22] Van den Hoogen F, Khanna D, Fransen J, et al. 2013 Classification Criteria for Systemic Sclerosis: An American College of Rheumatology/European League Against Rheumatism Collaborative Initiative. Arthritis Rheum. 2013;65:2737-2747. doi: 10.1002/art.38098. [23] Shiboski CH, Shiboski SC, Seror R, et al. 2016 American College of Rheumatology/European League Against Rheumatism Classification Criteria for Primary Sjögren's Syndrome: A Consensus and Data-Driven Methodology Involving Three International Patient Cohorts. Ann Rheum Dis. 2017;76:9-16. doi: 10.1136/annrheumdis-2016-210571.

[24] LeRoy EC, Black C, Fleischmajer R, et al. Scleroderma (Systemic Sclerosis) Classification, Subsets and Pathogenesis. J Rheumatol. 1988;15:202-205.
[25] Wolfe F, Clauw DJ, Fitzcharles MA, et al. 2016 Revisions to the 2010/2011 Fibromyalgia Diagnostic Criteria. Semin Arthritis Rheum. 2016;46:319-329. doi: 10.1016/j.semarthrit.2016.08.012.

[26] Maiani S, Sanavio E. Semantics of Pain in Italy: The Italian Version of the McGill Pain Questionnaire. Pain. 1985;22:399-405.

[27] Cuzdan N, Turk I, Sarpel T, et al. Neuropathic Pain: Is It An Underestimated Symptom in Systemic Sclerosis? Clin Rheumatol. 2018;37:1845-1851. doi: 10.1007/s10067-018-4107-1.

28. Ostojic P, Knezevic-Apostolski S, Djurovic N, et al. Neurological and Electroneurography Findings in Patients with Systemic Sclerosis and Symptoms of Neuropathic Pain in Extremities. Acta Neurol Belg. 2018;2018. doi: 10.1007/s13760-018-1048-z.

[29] Sousa-Neves J, Cerqueira M, Santos-Faria D, et al. Neuropathic Pain in Systemic Sclerosis Patients: A Cross-Sectional Study. Reumatol Clín. 2019;15:e99-e101. doi: 10.1016/j.reuma.2017.12.010.

[30] Varjú C, Péntek M, Lóránd V, et al. Musculoskeletal Involvement in Systemic Sclerosis: An Unexplored Aspect of the Disease. J Scleroderma Relat Disord. 2017;2:19-32. doi: 10.5301/ jsrd.5000228.

[31] Larrosa Pardo F, Bondesson E, Schelin MEC, et al. A Diagnosis of Rheumatoid Arthritis, Endometriosis or IBD is Associated with Later Onset of Fibromyalgia and Chronic Widespread Pain. Eur J Pain. 2019;23:1563-1573. doi: 10.1002/ejp.1432.

[32] Lo Giudice A, Brewer I, Leonardi R, et al. Pain Threshold and Temporomandibular Function in Systemic Sclerosis: Comparison with Psoriatic Arthritis. Clin Rheumatol. 2018;37:1861-1867. doi: 10.1007/s10067-018-4028-z.

[33] Ostojic P, Jankovic K, Djurovic N, et al. Common Causes of Pain in Systemic Sclerosis: Frequency, Severity, and Relationship to Disease Status, Depression, and Quality of Life. Pain Manag Nurs. 2019;20(4):331-336. doi: 10.1016/j.pmn.2019.02.006. 